\title{
Rotationally-Quasi-Invariant Measures on the Dual of a Hilbert Space
}

\author{
By \\ Hiroaki SHIMOMURA*
}

\section{$\S 1$. Introduction}

Let $H$ be a real Hilbert space equipped with the scalar product $\langle\cdot, \cdot\rangle_{H}$ and the norm $\|\cdot\|_{H}$. And let $H^{a}$ be the algebraic dual space of $H$. We consider a probability measure $\mu$ defined on the $\sigma$-field $\mathfrak{B}$ generated by cylinder sets of $H^{a}$. That is, $\mathfrak{B}$ is the minimal $\sigma$-field with which all functions $f_{h}(h \in H) ; x \in H^{a}$ $\mapsto x(h) \in \mathbb{R}$ are measurable. Let $O(H)$ be the group of all orthogonal operators on $H$. Then for each $U \in O(H)$ its algebraic transpose ${ }^{t} U$ is a measurable transformation on $\left(H^{a}, \mathfrak{B}\right)$. So we define $\mu_{U}$ as $\mu_{U}(B)=\mu\left({ }^{1} U^{-1}(B)\right)$ for all $B \in \mathfrak{B}$.

\section{Definition}

(a) $\mu$ is said to be rotationally-invariant, if $\mu_{U}=\mu$ holds for all $U \in O(H)$.

(b) $\mu$ is said to be rotationally-quasi-invariant, if $\mu_{U} \simeq \mu$ ( $\mu_{U}$ and $\mu$ are absolutely continuous with each other.) holds for all $U \in O(H)$.

It is well-known that rotationally-invariant measures are characterized as suitable sums of canonical Gaussian measures in terms of the variance parameter. (See, [2].) On the other hand, up to the present time the study of quasi-invariant measures is rather neglected. In this paper, we shall consider such measures and show in Theorem 2 that for any rotationally-quasi-invariant measure $\mu$, there exists a rotationally-invariant measure which is equivalent with $\mu$. First in $\$ 2$ we consider probability measures on $\mathbb{R}^{\infty}$ to discuss the rotational-quasiinvariance, and prove a version of the above statement. The proof of the main theorem will be carried out in $\S 3$.

Communicated by S. Matsuura, June 7, 1984.

* Department of Mathematics, Fukui University, Fukui 910, Japan. 


\section{§2. Rotationally-Quasi-Invariant Measures on $\boldsymbol{R}^{\infty}$}

Let $\boldsymbol{R}^{\infty}$ be the countable direct-product of $\boldsymbol{R}$ and put $\boldsymbol{R}_{0}^{\infty}=\left\{x=\left(x_{1}, \ldots, x_{n}\right.\right.$, $\ldots) \in \boldsymbol{R}^{\propto} \mid x_{n}=0$ except finite numbers of $\left.n\right\}$. Next, let $U$ be a one-to-one onto linear operator on $\boldsymbol{R}_{0}^{\infty}$ which is extended to an orthogonal operator on $l^{2}$. The group $G$ of all such $U$ 's will play an essential role in our discussions. Let $e_{n}=(0, \ldots, 0, \stackrel{n}{1}, 0, \ldots)(n=1,2, \ldots)$ be the canonical base on $\boldsymbol{R}_{0}^{\infty}$. Among subgroups of $G$, we shall take following important groups $O(n), O(\infty)$ and $O(n)^{\perp}$, $O(n)=\left\{U \in G \mid U e_{j}=e_{j}\right.$ for all $\left.j>n\right\}, O(\infty)=\cup_{n=1}^{\infty} O(n)$ and $O(n)^{\perp}=\{U \in O(\infty) \mid$ $U e_{j}=e_{j}$ for $\left.1 \leqq j \leqq n\right\}$. Clearly, we have $G \supset O(\infty) \supset O(n)^{\perp} \supset O(n+1)^{\perp}$. Now consider a probability measure $\mu$ on the usual Borel field $\mathfrak{B}\left(\boldsymbol{R}^{\infty}\right)$ on $\boldsymbol{R}^{\infty}$. Since for each $U \in G$, its transpose ${ }^{t} U$ (for the duality of $\boldsymbol{R}_{0}^{\infty}$ and $\boldsymbol{R}^{\infty}$ ) is a measurable transformation on $\boldsymbol{R}^{\infty}$, so $\mu_{U}$ is defined as before. If $\mu_{U} \simeq \mu$ holds for all $U \in G$ $\left(\mu_{U}=\mu\right.$ holds for all $\left.U \in O(\infty)\right), \mu$ is said to be rotationally- $G$-quasi-invariant (rotationally- $O(\infty)$-invariant), respectively. We begin with a following fundamental lemma.

Lemma 1. Let $\mu$ be rotationally-G-quasi-invariant and put

$$
\varepsilon_{n}=\sup _{U \in \boldsymbol{O}(n)} \int\left|\frac{d \mu_{U}}{d \mu}(x)-1\right| d \mu(x) .
$$

Then w'e have $\lim _{n} \varepsilon_{n}=0$.

Proof. As $\left\{\varepsilon_{n}\right\}$ is monotone decreasing, $\lim _{n} \varepsilon_{n}=\varepsilon$ exists at any rate. Assume that $\varepsilon>0$. Then there exists $U_{1} \in O(1)^{\perp}$ such that $\int\left|\frac{d \mu_{U_{1}}}{d \mu}(x)-1\right| d \mu(x)>\frac{\varepsilon}{2}$. From the definition of $O(1)^{\perp}, U_{1}$ belongs to $O\left(n_{2}\right)$ for some $n_{2}$. Without loss of generality we can assume that $n_{2}>1 \equiv n_{1}$. Next replacing $1=n_{1}$ by $n_{2}$ (noting $\varepsilon_{n_{2}}>\varepsilon / 2$ ), we repeat this procedure, and so on. Then as it is easily seen, a sequence $n_{1}<\cdots<n_{k}<\cdots$ and $U_{k} \in O\left(n_{k}\right)^{\perp} \cap O\left(n_{k+1}\right)$ are defined inductively such that

$$
\int\left|\frac{d \mu_{U_{k}}}{d \mu}(x)-1\right| d \mu(x)>\frac{\varepsilon}{2} \quad \text { for all } k
$$

Since $O\left(n_{k}\right)^{\perp} \cap O\left(n_{k+1}\right)$ is regarded as the orthogonal group on $\boldsymbol{R}^{n_{k+1}-n_{k}}$, it is compact in the natural topology. Hence the direct-product $K \equiv \prod_{k=1}^{\infty} O .\left(n_{k}\right)^{\perp}$ $\cap O\left(n_{k+1}\right)$ is again a compact group. Naturally, each element $W=\left(W_{1}, \ldots\right.$, 
$\left.W_{h}, \ldots\right) \in K$ acts on $\mathbb{R}_{0}^{\infty}$ as $\tilde{W} ; x=\sum_{j=1}^{\infty} x_{j} e_{j} \mapsto x_{1} e_{1}+\sum_{h=1}^{\infty} W_{k}\left(x_{n_{k}+1} e_{n_{k}+1}+\cdots\right.$ $\left.+x_{n_{k+1}} e_{n_{k+1}}\right)$. It is obvious that $\tilde{W} \in G$ for all $W \in K$. Put $\tilde{\mu}(B)=\int_{W \in K} \mu_{W}(B) d W$, where $d W$ is the normalized Haar measure of $K . \tilde{\mu}$ is invariant under the actions of $\tilde{W}(W \in K)$, especially $\tilde{\mu}=(\tilde{\mu})_{U_{h}}$, and $\tilde{\mu} \simeq \mu$ holds in virtue of $G$-quasiinvariance of $\mu$. Now $\frac{d \mu_{U_{k}}}{d \tilde{\mu}}(x)=\frac{d \mu}{d \tilde{\mu}}\left({ }^{t} U_{k}^{-1} x\right)$ holds for $\tilde{\mu}$-a.e. $x$, because for all $B \in \mathfrak{B}\left(\boldsymbol{R}^{\infty}\right)$ we have $\mu_{U_{k}}(B)=\int_{t_{U_{k}^{-1}}(B)} \frac{d \mu}{d \tilde{\mu}}(x) d \tilde{\mu}(x)=\int_{B} \frac{d \mu}{d \tilde{\mu}}\left({ }^{t} U_{k}^{-1} x\right) d \tilde{\mu}(x)$. It follows from (1) that

$$
\int\left|\frac{d \mu}{d \tilde{\mu}}\left({ }^{1} U_{k}^{-1} x\right)-\frac{d \mu}{d \tilde{\mu}}(x)\right| d \tilde{\mu}(x)>\frac{\varepsilon}{2} \quad \text { for all } \quad k .
$$

In this step, we take an $f \in L_{\tilde{\mu}}^{1}$ such that $\int\left|\frac{d \mu}{d \tilde{\mu}}(x)-f(x)\right| d \tilde{\mu}(x)<\frac{\varepsilon}{8}$ and $f$ depends on only finite numbers of coordinates, say $x_{1}, \ldots, x_{s}$. Thus if $n_{k} \geqq s$, we have $f(x)=f\left({ }^{t} U_{k}^{-1} x\right)$. Consequently for $n_{k} \geqq s$ we have

$$
\begin{aligned}
& \int\left|\frac{d \mu}{d \tilde{\mu}}\left({ }^{t} U_{k}^{-1} x\right)-\frac{d \mu}{d \tilde{\mu}}(x)\right| d \tilde{\mu}(x) \leqq \int\left|\frac{d \mu}{d \tilde{\mu}}\left({ }^{t} U_{k}^{-1} x\right)-f\left({ }^{t} U_{h}^{-1} x\right)\right| d \tilde{\mu}(x) \\
& \quad+\int\left|\frac{d \mu}{d \tilde{\mu}}(x)-f(x)\right| d \tilde{\mu}(x)<\frac{\varepsilon}{8}+\frac{\varepsilon}{8}=\frac{\varepsilon}{4} .
\end{aligned}
$$

However it contradicts to (2).

We note that

$$
\int\left|\frac{d \mu_{U}}{d \mu}(x)-1\right| d \mu(x)=2 \sup \left\{\left|\mu_{U}(B)-\mu(B)\right| \mid B \in \mathfrak{B}\left(\mathbb{R}^{\infty}\right)\right\} .
$$

Now we shall proceed to the definition of the limiting measure $\mu_{\omega}$ of $\mu$. Let $E, F$ be Borel sets of $\mathbb{R}^{m}, \boldsymbol{R}^{l}$ respectively, and put $P_{m} ; x \in \mathbb{R}^{\infty} \mapsto\left(x_{1}, \ldots, x_{m}\right) \in \mathbb{R}^{m}$. Applying Lemma 1,

$$
\lim _{n} \mu\left(x \in \mathbb{R}^{\infty} \mid\left(x_{1}, \ldots, x_{m}\right) \in E,\left(x_{n+1}, \ldots, x_{n+l}\right) \in F\right) \equiv \mu_{l}\left(F \mid P_{m}^{-1}(E)\right)
$$

exists for all $E, F$. Because for $n^{\prime} \geq n \geq m$ choose $U_{n \cdot n^{\prime}} \in O(n)^{\perp}$ such that $U_{n, n^{\prime}} \cdot e_{n+1}=e_{n^{\prime}+1}, \ldots, U_{n \cdot n^{\prime}} e_{n+l}=e_{n^{\prime}+l}$. Then we have

$$
\begin{aligned}
\mu_{U_{n, n^{\prime}}}\left(x \mid\left(x_{1}, \ldots, x_{m}\right)\right. & \left.\in E,\left(x_{n+1}, \ldots, x_{n+l}\right) \in F\right) \\
& =\mu\left(x \mid\left(x_{1}, \ldots, x_{m}\right) \in E,\left(x_{n^{\prime}+1}, \ldots, x_{n^{\prime}+l}\right) \in F\right) .
\end{aligned}
$$

Hence 


$$
\begin{aligned}
& 2 \mid \mu\left(x \mid\left(x_{1}, \ldots, x_{m}\right) \in E,\left(x_{n^{\prime}+1}, \ldots, x_{n^{\prime}+l}\right) \in F\right) \\
& -\mu\left(x \mid\left(x_{1}, \ldots, x_{m}\right) \in E,\left(x_{n+1}, \ldots, x_{n+l}\right) \in F\right) \mid \\
& \leqq \sup _{U \in O(n)} \int\left|\frac{d \mu_{U}}{d \mu}(x)-1\right| d \mu(x)=\varepsilon_{n} \quad \text { for } \quad n^{\prime} \geq n \geq m .
\end{aligned}
$$

Therefore they form a Cauchy sequence. It is obvious that for $l<l^{\prime}, \mu_{l^{\prime}}(F \times$ $\left.\boldsymbol{R}^{l^{\prime}-l} \mid P_{m}^{-1}(E)\right)=\mu_{l}\left(F \mid P_{m}^{-1}(E)\right)$. Consequently $\left\{\mu_{l}\left(\cdot \mid P_{m}^{-1}(E)\right)\right\}_{l}$ forms a consistent family of measures on $\left\{\boldsymbol{R}^{l}\right\}_{l}$ by natural projections, and a measure $\mu_{\omega}\left(\cdot \mid P_{m}^{-1}(E)\right)$ is defined on $\mathfrak{B}\left(\boldsymbol{R}^{\infty}\right)$ such that $\mu_{o}\left(P_{l}^{-1}(F) \mid P_{m}^{-1}(E)\right)=\mu_{l}\left(F \mid P_{m}^{-1}(E)\right)$. Especially, we simply write $\mu_{\omega}(\cdot)$ instead of $\mu_{\omega}\left(\cdot \mid R^{\infty}\right)$.

Lemma 2. For any $m$ and for any Borel set $E \subset \boldsymbol{R}^{m}, \mu_{\omega}\left(\cdot \mid P_{m}^{-1}(E)\right)$ is $O(\infty)$-invariant

Proof. For the proof it is necessary and sufficient to show $\mu_{l}\left(\cdot \mid P_{m}^{-1}(E)\right)$ is $O\left(\boldsymbol{R}^{l}\right)$-invariant for all $l$. Let $U \in O\left(\boldsymbol{R}^{l}\right)$. Then for each $n \geq m$ we can take an $U_{n} \in O(n)^{\perp} \cap O(n+l)$ such that $\mu_{U_{n}}\left(x \mid\left(x_{1}, \ldots, x_{m}\right) \in E,\left(x_{n+1}, \ldots, x_{n+l}\right) \in F\right)$ $=\mu\left(x \mid\left(x_{1}, \ldots, x_{m}\right) \in E,\left(x_{n+1}, \ldots, x_{n+l}\right) \in U F\right)$, for all Borel sets $F \subset \boldsymbol{R}^{l}$. Hence by Lemma 1, $\quad \mu\left(x \mid\left(x_{1}, \ldots, x_{m}\right) \in E,\left(x_{n+1}, \ldots, x_{n+l}\right) \in U F\right)-\mu\left(x \mid\left(x_{1}, \ldots, x_{m}\right) \in E\right.$, $\left.\left(x_{n+1}, \ldots, x_{n+l}\right) \in F\right) \mid \leqq 2^{-1} \varepsilon_{n} \rightarrow 0(n \rightarrow \infty)$.

This implies that $\mu_{l}\left(U F \mid P_{m}^{-1}(E)\right)=\mu_{l}\left(F \mid P_{m}^{-1}(E)\right)$.

Q.E. D.

By the above Lemma, $\mu_{\omega}\left(\cdot \mid P_{m}^{-1}(E)\right)$ is represented by a suitable sum of canonical Gaussian measures $g_{v}$ with mean 0 and variance $v$ on $\mathfrak{B}\left(\boldsymbol{R}^{\infty}\right)$. (See, for example [1].) Next let $\mathfrak{B}^{n}$ be a minimal $\sigma$-field on $\boldsymbol{R}^{\infty}$ with which all the coordinate functions $x_{n+1}, \ldots, x_{n+k}, \ldots$ are measurable and put $\mathfrak{B}_{\infty}=\cap_{n=1}^{\infty} \mathfrak{B}^{n}$.

Lemma 3. For any $m$ and for any Borel set $E \subset \boldsymbol{R}^{m}, \mu_{\omega}\left(B \mid P_{m}^{-1}(E)\right)=$ $\mu\left(B \cap P_{m}^{-1}(E)\right)$ holds for all $B \in \mathfrak{B}_{\infty}$.

Proof. Letting $n^{\prime} \rightarrow \infty$ in (3), we have

$$
\left|\mu_{\omega}\left(P_{l}^{-1}(F) \mid P_{m}^{-1}(E)\right)-\mu\left(x \mid\left(x_{1}, \ldots, x_{m}\right) \in E,\left(x_{n+1}, \ldots, x_{n+l}\right) \in F\right)\right| \leqq 2^{-1} \varepsilon_{n}
$$

for all $n \geqq m$. By $O(\infty)$-invariance of $\mu_{c v}\left(\cdot \mid P_{m}^{-1}(E)\right)$, the above inequality becomes, $\quad \mid \mu_{\omega}\left(\left(x_{n+1}, \ldots, x_{n+l}\right) \in F \mid P_{m}^{-1}(E)\right)-\mu\left(x \mid\left(x_{1}, \ldots, x_{m}\right) \in E,\left(x_{n+1}, \ldots, x_{n+l}\right)\right.$ $\in F) \mid \leqq 2^{-1} \varepsilon_{n}$ for all $n \geqq m$. As the right hand does not depend on $l$, so for all $n \geqq m$ and for all $B \in \mathfrak{B}^{n}$ we have $\left|\mu_{\omega}\left(B \mid P_{m}^{-1}(E)\right)-\mu\left(P_{m}^{-1}(E) \cap B\right)\right| \leqq 2^{-1} \varepsilon_{n}$. Especially for any $B \in \mathfrak{B}_{\infty}$ it holds independently on $n$. So the proof is complete, letting $n \rightarrow \infty$.

Q.E.D. 
In order to observe the explicit form of $\mu_{\omega}\left(\cdot \mid P_{m}^{-1}(E)\right)$, we use a family of conditional probability measures $\left\{\mu^{x}\right\}_{x \in \mathbb{R}^{\infty}}$ of $\mu$ with respect to $\mathfrak{B}_{\infty}$. For $\left\{\mu^{x}\right\}_{x \in R^{\infty}}$, it is well-known that

(a) for every $x \in \mathbb{R}^{\alpha}, \mu^{x}$ is a probability measure on $\mathfrak{B}\left(\mathbb{R}^{\infty}\right)$,

(b) for a fixed $E \in \mathfrak{B}\left(\mathbb{R}^{\infty}\right), \mu^{x}(E)$ is a $\mathfrak{B}_{\infty}$-measurable function of $x \in \mathbb{R}^{\infty}$,

(c) $\mu(E \cap B)=\int_{B} \mu^{x}(E) d \mu(x)$ for all $E \in \mathfrak{B}\left(\mathbb{R}^{\infty}\right)$ and for all $B \in \mathfrak{B}_{\infty}$.

Now let $B \in \mathfrak{B}_{\alpha}$. Then by Lemma 3 ,

$$
\mu_{\omega}\left(B \mid P_{m}^{-1}(E)\right)=\mu\left(B \cap P_{m}^{-1}(E)\right)=\int_{B} \mu^{x}\left(P_{m}^{-1}(E)\right) d \mu(x)=\int_{B} \mu^{x}\left(P_{m}^{-1}(E)\right) d \mu_{\omega}(x) .
$$

By the way, for a fixed $E, \lambda_{E}(A)=\int_{A} \mu^{x}\left(P_{m}^{-1}(E)\right) d \mu_{\omega}(x)$ is an $O(\infty)$-invariant measure in virtue of (b) and of $O(\infty)$-invariance of $\mu_{\omega}$. As the form of $O(\infty)$ invariant measure is completely determined on $\mathfrak{B}_{\infty}$, (See, [1].) it follows from the above that $\lambda_{E}(\cdot)=\mu_{\omega}\left(\cdot \mid P_{m}^{-1}(E)\right)$. Consequently,

Lemma 4. Let $\left\{\mu^{x}\right\}_{x \in \mathbb{R}^{\infty}}$ be the conditional probability measures of $\mu$ with respect to $\mathfrak{B}_{\infty}$. Then we have

$$
\mu_{\omega}\left(B \mid P_{m}^{-1}(E)\right)=\int_{B} \mu^{x}\left(P_{m}^{-1}(E)\right) d \mu_{\omega}(x), \quad \text { for all } B \in \mathfrak{B}\left(\mathbb{R}^{\infty}\right) .
$$

Here we shall add brief results for $\mu_{\omega}$. (The proofs are obvious.)

1. $\mu=\mu_{\omega}$, if $\mu$ is $O(\infty)$-invariant.

2. If $\mu$ is a convex sum of two rotationally-G-quasi-invariant measures $\mu^{1}$ and $\mu^{2}$, then $\mu_{\omega}$ is the same sum of $\mu_{\omega}^{1}$ and $\mu_{\omega}^{2}$.

We shall prove $\mu \simeq \mu_{\omega}$ in the remainder part of this section. Now consider an isometric operator $S$ on $l^{2}, S e_{1}=e_{2}, \ldots, S e_{n}=e_{2 n}, \ldots$. Corresponding to $S$, we take $U_{n} \in O(2 n)$ for each $n$ such that $U_{n} e_{1}=e_{2}, U_{n} e_{2}=e_{4}, \ldots, U_{n} e_{n}=e_{2 n}$, $U_{n} e_{n+1}=e_{1}, \quad U_{n} e_{n+2}=e_{3}, \ldots, U_{n} e_{n+k}=e_{2 k-1}, \ldots, U_{n} e_{2 n}=e_{2 n-1}$. Since $U_{l} e_{j}=$ $U_{m} e_{j}(j=1, \ldots, n)$ for $n \leqq l \leqq m$, we have $U_{m}^{-1} U_{l} \in O(n)^{\perp}$. It follows that $\sup \left\{\left|\mu\left({ }^{t} U_{m}(E)\right)-\mu\left({ }^{t} U_{l}(E)\right)\right| \mid E \in \mathfrak{B}\left(\boldsymbol{R}^{\infty}\right)\right\}=\sup \left\{\left|\mu\left({ }^{t} U_{m}{ }^{t} U_{l}^{-1}(E)\right)-\mu(E)\right| \mid E \in\right.$ $\left.\mathfrak{B}\left(\boldsymbol{R}^{\infty}\right)\right\} \leqq 2^{-1} \varepsilon_{n} . \quad$ Therefore by Lemma $1, \lim \mu\left({ }^{t} U_{n}(E)\right) \equiv \mu_{S}(E)$ exists for all $E \in \mathfrak{B}\left(\mathbb{R}^{\infty}\right)$. It is obvious that $\mu_{s} \lesssim \mu$. Further putting $\mathscr{U}_{\infty}=\left\{\left.E \in \mathfrak{B}\left(\mathbb{R}^{\infty}\right)\right|^{t} U E\right.$ $=E$ for $\left.{ }^{\forall} U \in O(\infty)\right\}, \mu_{S}=\mu$ holds on $\mathfrak{A}_{\infty}$. In order to observe $\mu_{\mathrm{S}}$, we put $p ; x=$ $\left(x_{1}, \ldots, x_{n}, \ldots\right) \in \mathbb{R}^{\infty} \mapsto\left(x_{1}, x_{3}, \ldots, x_{2 n-1}, \ldots\right) \in \mathbb{R}^{\infty}, q ; x=\left(x_{1}, \ldots, x_{n}, \ldots\right) \in \mathbb{R}^{\infty} \mapsto\left(x_{2}\right.$, $\left.x_{4}, \ldots, x_{2 n}, \ldots\right) \in \mathbb{R}^{\infty}$, and $T ; x \in \mathbb{R}^{\infty} \mapsto(p(x), q(x)) \in \mathbb{R}^{\infty} \times \mathbb{R}^{\infty}$. If $E, F$ are Borel 
sets of $\boldsymbol{R}^{m}, \mu_{S}\left(p^{-1}\left(P_{m}^{-1}(F)\right) \cap q^{-1}\left(P_{m}^{-1}(E)\right)=\mu_{S}\left(x \mid\left(x_{1}, x_{3}, \ldots, x_{2 m-1}\right) \in F,\left(x_{2}, x_{4}, \ldots\right.\right.\right.$, $\left.\left.x_{2 m}\right) \in E\right)=\lim _{n} \mu\left(x \mid\left(x_{1}, x_{2}, \ldots, x_{m}\right) \in E,\left(x_{n+1}, x_{n+2}, \ldots, x_{n+m}\right) \in F\right)=\mu_{m}\left(F \mid P_{m}^{-1}(E)\right)$ $=\mu_{\omega}\left(P_{m}^{-1}(F) \mid P_{m}^{-1}(E)\right)=\int_{P_{m}^{-1}(F)} \mu^{x}\left(P_{m}^{-1}(E)\right) d \mu_{\omega}(x)$. It follows that

$$
\mu_{S}\left(p^{-1}\left(B_{1}\right) \cap q^{-1}\left(B_{2}\right)\right)=\int_{B_{1}} \mu^{x}\left(B_{2}\right) d \mu_{\omega}(x) \quad \text { for all } \quad B_{1}, B_{2} \in \mathfrak{B}\left(\boldsymbol{R}^{\infty}\right) .
$$

Hence

$$
\begin{aligned}
& T \mu_{S}\left(B_{1} \times B_{2}\right)=\mu_{S}\left(p^{-1}\left(B_{1}\right) \cap q^{-1}\left(B_{2}\right)\right) \\
& \quad=\int \delta_{x}\left(B_{1}\right) \mu^{x}\left(B_{2}\right) d \mu_{\omega}(x)=\int\left(\delta_{x} \times \mu^{x}\right)\left(B_{1} \times B_{2}\right) d \mu_{\omega}(x) .
\end{aligned}
$$

Consequently, we have

$$
T \mu_{S}(B)=\int\left(\delta_{x} \times \mu^{x}\right)(B) d \mu_{\omega}(x) \quad \text { for all } \quad B \in \mathfrak{B}\left(\boldsymbol{R}^{\infty} \times \boldsymbol{R}^{\infty}\right) .
$$

Here we consider translational-quasi-invariance of $\mu_{S}$. (For these notions we refer [1], [3] or [4].)

Lemma 5. If $\mu_{\omega}$ is translationally- ${ }^{2}$-quasi-invariant (equivalently the Dirac term of $\mu_{\omega}$ is dropped), then for any $h \in l^{2}$ there exists $B_{h} \in \mathfrak{B}_{\infty}$ with $\mu_{\omega}\left(B_{h}\right)=1$ such that $\mu^{x}=\mu^{x+h}$ for all $x \in B_{h}$.

Proof. Since $\mu_{\omega}([B-h] \ominus B)=0$ for all $B \in \mathfrak{B}_{\infty}$ (See, [1]), the same holds for $\mu$ by Lemma 3. Especially, putting $\mu_{h}(E)=\mu(E-h)$ for all $E \in \mathfrak{B}\left(\boldsymbol{R}^{\infty}\right)$, $\mu_{h}=\mu$ holds on $\mathfrak{B}_{\infty}$. It follows that for any $B \in \mathfrak{B}_{\infty}$ and for any $E \in \mathfrak{B}\left(\boldsymbol{R}^{\infty}\right)$,

$$
\begin{aligned}
\mu(E \cap B) & =\int_{B} \mu^{x}(E) d \mu(x)=\int_{B} \mu^{x}(E) d \mu_{h}(x) \\
& =\int_{B-h} \mu^{x+h}(E) d \mu(x)=\int_{B} \mu^{x+h}(E) d \mu(x) .
\end{aligned}
$$

As $\mu^{x}(E)$ and $\mu^{x+h}(E)$ are both $\mathfrak{B}_{\infty}$-measurable functions, so $\mu^{x}(E)=\mu^{x+h}(E)$ holds for $\mu$-a.e.x. Take a countable algebra $\mathscr{F}$ generating $\mathfrak{B}\left(\boldsymbol{R}^{\infty}\right)$ and put $B_{h}=\cap_{S_{\in F}}\left\{x \mid \mu^{x}(S)=\mu^{x+h}(S)\right\}$. It is clear that $B_{h} \in \mathfrak{B}_{\infty}, \quad 1=\mu\left(B_{h}\right)=\mu_{\omega}\left(B_{h}\right)$ and $\mu^{x}=\mu^{x+h}$ holds for all $x \in B_{h}$.

Lemma 6. If $\mu_{\omega}$ is translationally- $l^{2}$-quasi-invariant, then we have $\left(\mu_{S}\right)_{h} \simeq \mu_{S}$ for all $\hat{h}=h_{1} e_{1}+h_{2} e_{3}+\cdots+h_{n} e_{2 n-1}+\cdots, \sum_{n=1}^{\infty} h_{n}^{2}<\infty$.

Proof. It is enough to show that $T \mu_{S}$ is translationally-quasi-invariant for all $(h, 0) \in l^{2} \times \boldsymbol{R}^{\infty}$. Using (4) and Lemma 5 , it is assured as follows.

$$
T \mu_{S}(B)=0 \Longleftrightarrow \int\left(\delta_{x} \times \mu^{x}\right)(B) d \mu_{\omega}(x)=0 \Longleftrightarrow
$$




$$
\begin{aligned}
& \int \mu^{x}(y \mid(0, y) \in B-(x, 0)) d \mu_{\omega}(x)=0 \Longleftrightarrow \\
& \int \mu^{x+h}(y \mid(0, y) \in B-(h, 0)-(x, 0)) d \mu_{\omega}(x)=0 \Longleftrightarrow \\
& \int_{B_{h}} \mu^{x}(y \mid(0, y) \in B-(h, 0)-(x, 0)) d \mu_{\omega}(x)=0 \Longleftrightarrow T \mu_{S}(B-(h, 0))=0
\end{aligned}
$$

Next we consider the effect of the Dirac term of $\mu_{\omega}$. The following three cases are possible.

(a) $\mu(\{0\})=1$. In this case $\mu=\mu_{\omega}=\delta_{0}$, so it is nothing to prove.

(b) $\mu(\{0\})=0$.

(c) $0<\mu(\{0\})<1$. Put $\mu^{1}(E)=\frac{\mu\left(E \cap\{0\}^{c}\right)}{\mu\left(\{0\}^{c}\right)}$ for all $E \in \mathfrak{B}\left(\mathbb{R}^{\infty}\right)$.

Then $\mu^{1}$ is rotationally-G-quasi-invariant. And we have

$$
\mu=\mu(\{0\}) \delta_{0}+\mu\left(\{0\}^{c}\right) \mu^{1} \quad \text { and } \quad \mu_{\omega}=\mu(\{0\}) \delta_{0}+\mu\left(\{0\}^{c}\right) \mu_{\omega}^{1} .
$$

Thus for the proof of $\mu \simeq \mu_{\omega}$, it is sufficient to consider the case (b). Now let $\mu(\{0\})=0$, and put $N_{n}=\left\{x \in \boldsymbol{R}^{\infty} \mid x_{n}=0\right\}$ for each $n$. We wish to show $\mu\left(N_{1}\right)=0$, equivalently $\mu\left(N_{n}\right)=0$ for all $n$. Suppose that it would be false. Since $0=$ $\mu(\{0\})=\lim \mu\left(N_{1} \cap N_{2} \cap \cdots \cap N_{n}\right), \mu\left(N_{1}\right)>\mu\left(N_{1} \cap \cdots \cap N_{n}\right)$ holds for sufficiently large $n$. It follows that $\mu\left(N_{1} \cap N_{k}^{c}\right)>0$ for some $k \geqq 2$, equivalently $\mu\left(N_{1} \cap N_{2}^{c}\right)$ $>0$. Take $U_{\theta} \in O(2), \quad U_{\theta} e_{1}=\cos \theta e_{1}+\sin \theta e_{2}, \quad U_{\theta} e_{2}=-\sin \theta e_{1}+\cos \theta e_{2}$. As we have ${ }^{t} U_{\theta}^{-1}\left(N_{1} \cap N_{2}^{c}\right)=\left\{x \in \mathbb{R}^{\infty} \mid x_{1} \cos \theta+x_{2} \sin \theta=0, \quad-x_{1} \sin \theta+x_{2} \cos \theta\right.$ $\neq 0\}$, so they are mutually disjoint for different $\theta \in[0, \pi)$. Hence we conclude that $0=\mu\left({ }^{t} U_{\theta}^{-1}\left(N_{1} \cap N_{2}^{c}\right)\right)=\mu_{U_{\theta}}\left(N_{1} \cap N_{2}^{c}\right)$. However it contradicts to $\mu\left(N_{1} \cap\right.$ $\left.N_{2}^{c}\right)>0$. From $\mu\left(N_{n}\right)=0$, it follows that $\mu_{\omega}\left(N_{1}\right)=\lim _{n} \mu\left(N_{n}\right)=0$ and therefore $\mu_{\omega}(\{0\}) \leqq \mu_{\omega}\left(N_{1}\right)=0$. From these arguments,

Lemma 7. If $\mu$ has no Dirac term, then so is $\mu_{\omega}$.

Hereafter assume that $\mu(\{0\})=0$. Then we take a probability measure $\sigma$ on $\mathfrak{B}\left(\boldsymbol{R}^{\infty}\right)$ which is translationally- $\boldsymbol{R}_{0}^{\infty}$-quasi-invariant and $\sigma\left(l^{2}\right)=1$. The convolution $\mu * \sigma$ and $\mu_{\omega}$ are both translationally- $\mathbb{R}_{0}^{\infty}$-quasi-invariant, and for any $B \in \mathfrak{B}_{\infty}$,

$$
\mu * \sigma(B)=\int_{l^{2}} \mu(B-h) d \sigma(h)=\int_{l^{2}} \mu(B) d \sigma(h)=\mu(B)=\mu_{\omega}(B) .
$$

Since the equivalence classes of translationally- $\mathbb{R}_{0}^{\infty}$-quasi-invariant measures are completely determined on $\mathfrak{B}_{\infty}$, (See, [1]) we conclude that $\mu * \sigma \simeq \mu_{\omega}$. Using 
these results and the following last Lemma we prove that $\mu \simeq \mu_{\omega}$.

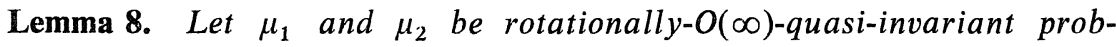
ability measures. Then for $\mu_{1} \lesssim \mu_{2}$, it is necessary and sufficient that $\mu_{1}$ $\lesssim \mu_{2}$ on $\mathfrak{P}_{\alpha}$.

Proof. The necessity is obvious. For the sufficiency, put $\mu=\frac{\mu_{1}+\mu_{2}}{2}$. Then there exists some $A \in \mathfrak{B}\left(\boldsymbol{R}^{\infty}\right)$ such that $\mu(A \cap B)=0 \Leftrightarrow \mu_{2}(B)=0$ for any $B \in \mathfrak{B}\left(\boldsymbol{R}^{\infty}\right)$. We claim that $A$ can be taken such as $A \in \mathfrak{A}_{\infty}$. Since we have $\mu_{2}\left(A^{c}\right)=0$, so $\mu_{2}\left({ }^{t} U A^{c}\right)=0$ and therefore $\mu\left(A \cap \cap^{t} U A^{c}\right)=0$. Replacing $U$ by $U^{-1}$, $\mu\left(A \Theta^{t} U A\right)=0$ holds for all $U \in O(\infty)$. Now using the indicator function $\chi_{A}$ of $A$ and the Haar measure $d U$ of $O(n)$, we put $g_{n}(x)=\int_{O(n)} \chi_{A}\left({ }^{t} U x\right) d U$. Then $g_{n}$ is $O(n)$-invariant and $g_{n}(x)=\chi_{A}(x)$ holds for $\mu$-a.e. $x$. Hence putting $\varlimsup_{n}$ $g_{n}(x)=g(x), g$ is $O(\infty)$-invariant and $g(x)=\chi_{A}(x)$ holds for $\mu$-a.e. $x$. Finally, put $\hat{A}=\left\{x \in \boldsymbol{R}^{\infty} \mid g(x)=1\right\}$. Then it is easily checked that $\hat{A} \in \mathfrak{A}_{\infty}$ and $\mu(A \ominus \hat{A})$ $=0$. Under the above preparation, let $\mu_{1} \lesssim \mu_{2}$ hold on $\mathfrak{A}_{\infty}$. Then we have $\mu_{1}\left(\hat{A}^{c}\right)=0 . \quad$ And if $\mu_{2}(E)=0$ for some $E \in \mathfrak{B}\left(\boldsymbol{R}^{\infty}\right)$, then we have $\mu(E \cap \hat{A})=0$ which implies $\mu_{1}(E \cap \hat{A})=0$. It follows that $\mu_{1}(E) \leqq \mu_{1}(E \cap \hat{A})+\mu_{1}\left(E \cap \hat{A}^{c}\right)=0$.

Q.E.D.

Theorem 1. For a rotationally-G-quasi-invariant measure $\mu$, we have $\mu \simeq \mu_{\omega}$.

Proof. By the preceding arguments, it may be assumed that $\mu(\{0\})=0$. First we shall show $\mu \lesssim \mu_{\omega}$. Let $A \in \mathfrak{R}_{\infty}$ and $\mu_{\omega}(A)=0$ which is equivalent to $\mu * \sigma(A)=0$. It implies that $\mu(A-h)=0$ for some $h=h_{1} e_{1}+\cdots+h_{n} e_{n}+\cdots \in l^{2}$. Take $\theta_{n}$ for each $n$ such that ${ }^{t} U^{-1} h=\sqrt{h_{1}^{2}+h_{2}^{2}} e_{1}+\cdots+\sqrt{h_{2 n-1}^{2}+h_{2 n}^{2}} e_{2 n-1}+\cdots$ holds for $U \in G$ defined by $U e_{2 n-1}=\cos \theta_{n} e_{2 n-1}+\sin \theta_{n} e_{2 n}, U e_{2 n}=-\sin \theta_{n} e_{2 n-1}$ $+\cos \theta_{n} e_{2 n}(n=1,2, \ldots)$. Since for any $U_{n} \in O(n)$, we have $W_{n}=U^{-1} U_{n} U \in$ $O(n+1)$, so ${ }^{t} U_{n}^{-1}{ }^{t} U^{-1}(A)={ }^{t} U^{-1}{ }^{t} W_{n}^{-1}(A)={ }^{t} U^{-1}(A)$ and therefore ${ }^{t} U^{-1}(A) \epsilon$ $\mathfrak{2}_{\infty}$. It follows from $\mu_{U}(A-h)=0$ that $\mu\left({ }^{t} U^{-1}(A)-{ }^{t} U^{-1} h\right)=0$ which implies $\mu_{S}\left({ }^{t} U^{-1}(A)-{ }^{t} U^{-1} h\right)=0$. By Lemma 6, we have $\mu_{S}\left({ }^{t} U^{-1}(A)\right)=0$. As $\mu=$ $\mu_{S}$ holds on $\mathfrak{A}_{\infty}$, so it holds $\mu\left({ }^{t} U^{-1}(A)\right)=0$, equivalently $\mu(A)=0$.

Next we shall show $\mu_{(i)} \lesssim \mu$. We use a representation of $\mu_{\omega}$ by a probability measure $P$ on $(0, \infty), \mu_{\omega}(B)=\int_{(0, \infty)} g_{v}(B) d P(v)$ for all $B \in \mathfrak{B}\left(\boldsymbol{R}^{\infty}\right)$. Put $r(x)=$ $\varlimsup_{N} \frac{1}{N} \sum_{n=1}^{N} x_{n}^{2}$. Then $r(x)$ is a $\mathfrak{B}_{\infty}$-measurable function and $g_{v}\left(r^{-1}(v)\right)=1$ by the law of large numbers. It follows that $\mu_{\omega}(x \mid r(x) \in(\alpha, \beta])=P((\alpha, \beta])$. By 
the way, $g_{v}$ takes only the values 1 or 0 on $\mathfrak{U}_{\infty}$, because $g_{v}$ is $O(\infty)$-ergodic. Now we put $B_{A}=r^{-1}\left\{v \mid g_{v}(A)=1\right\}$ for each $A \in \mathfrak{A}_{\infty}$. Then $g_{v}(A)=1$ implies $B_{A} \supset r^{-1}(v)$ and therefore $g_{v}\left(B_{A}\right)=1$. While, $g_{v}(A)=0$ implies $B_{A} \cap r^{-1}(v)=\varnothing$ and therefore $g_{v}\left(B_{A}\right)=0$. Consequently, $g_{v}\left(A \ominus B_{A}\right)=0$ for all $v$, hence we have $\mu_{\omega}\left(A \ominus B_{A}\right)=0$. We note the same holds for $\mu$, since we have seen $\mu_{\omega} \gtrsim \mu$. Now the proof follows from these arguments and Lemma 3. Let $A \in \mathfrak{P}_{\infty}$ and $\mu(A)=0$. Taking $B_{A} \in \mathfrak{B}_{\infty}$ as above, we have $0=\mu\left(B_{A}\right)=\mu_{\omega}\left(B_{A}\right)=\mu_{\omega}(A)$.

Q.E.D.

\section{§3. Rotationally-Quasi-Invariant Measures on $\mathbb{H}^{a}$}

In this section we prove the result announced in the introduction. Let $\mu$ be a rotationally-quasi-invariant probability measure on $\left(H^{a}, \mathfrak{B}\right)$. Take an arbitrary countably-infinite orthonormal system $f_{1}, f_{2}, \ldots, f_{n}, \ldots$ and put $\mu^{f}=T_{f} \mu$ by a map $T_{f} ; x \in H^{a} \mapsto\left(x\left(f_{1}\right), \ldots, x\left(f_{n}\right), \ldots\right) \in \mathbb{R}^{\infty}$. Then $\mu^{f}$ is a rotationally-Gquasi-invariant measure on $\mathfrak{B}\left(\mathbb{R}^{\infty}\right)$. Because taking an $\hat{U} \in O(H)$ for each $U \in G$ such that $\left\langle\hat{U} f_{k}, f_{j}\right\rangle_{H}=\left\langle U e_{k}, e_{j}\right\rangle_{1^{2}}(k, j=1,2, \ldots)$, we can assure that $T_{f}{ }^{t} \hat{U}=$ ${ }^{t} U T_{f}$. It follows that $\mu^{f}=T_{f} \mu \simeq T_{f}{ }^{t} \hat{U} \mu={ }^{t} U T_{f} \mu={ }^{t} U \mu^{f}$. Hence the limiting measure $\mu_{\omega}^{f}$ is defined, $\mu_{\omega,}^{f}(B)=\int_{[0, \infty)} g_{v}(B) d P^{f}(v)$ for all $B \in \mathfrak{B}\left(\mathbb{R}^{\infty}\right)$, and it holds $\mu^{f} \simeq \mu_{\omega}^{f}$ by Theorem 1. In order to observe that $P^{f}$ does not depend on the choice of $f_{1}, \ldots, f_{n}, \ldots$, we take another system $f_{1}^{\prime}, \ldots, f_{n}^{\prime}, \ldots$ We perform Schmidt's orthogonalization process for $f_{1}, f_{1}^{\prime}, \ldots, f_{n}, f_{n}^{\prime}, \ldots$ to obtain an orthonormal system $h_{1}, \ldots, h_{n}, \ldots$. It is clear that $f_{n}$ and $f_{n}^{\prime}$ are finite linear combinations of $h_{1}, \ldots, h_{n}, \ldots$, . We wish to show $P^{f}=P^{h}$. Now consider an operator $T$ on $\boldsymbol{R}_{0}^{\infty}$ such that $T e_{n}=\sum_{k=1}^{\infty}\left\langle f_{n}, h_{k}\right\rangle_{H} e_{k}$ for each $n$. (Actually it is a finite sum.) $T$ preserves $l^{2}$-norm as easily seen. Hence we have ${ }^{t} T \mu_{\omega}^{h}=\mu_{\omega}^{h}$. Further noting that $\left\{x \in \mathbb{R}^{\infty} \mid r\left({ }^{t} T x\right) \in(\alpha, \beta]\right\} \in \mathfrak{B}_{\infty}$ for $\alpha, \beta \in \mathbb{R}$, it follows that $P^{h}((\alpha, \beta])=$ $\mu_{\omega}^{h}\left(x \in \mathbb{R}^{\infty} \mid r(x) \in(\alpha, \beta]\right)=\mu_{\omega}^{h}\left(x \in \mathbb{R}^{\infty} \mid r\left({ }^{t} T x\right) \in(\alpha, \beta]\right)=\mu^{h}\left(x \in \mathbb{R}^{\infty} \mid r\left({ }^{t} T x\right) \in(\alpha, \beta]\right)$ $=\mu\left(x \in H^{a} \mid r\left({ }^{t} T T_{l} x\right) \in(\alpha, \beta]\right)=\mu\left(x \in H^{a} \mid r\left(T_{f} x\right) \in(\alpha, \beta]\right)=\mu^{f}\left(x \in \mathbb{R}^{\infty} \mid r(x) \in(\alpha, \beta]\right)$ $=P^{f}((\alpha, \beta])$. Similarly we have $P^{h}=P^{f^{\prime}}$. So putting $P=P^{f}=P^{f^{\prime}}$, a rotationally-invariant probability measure $v$ is defined on $\left(H^{a}, \mathfrak{B}\right), v(B)=\int_{[0, \infty)}$ $G_{v}(B) d P(v)$, for all $B \in \mathfrak{B}$, where $G_{v}$ is a canonical Gaussian measure on $\left(H^{a}, \mathfrak{B}\right)$ with mean 0 and variace $v$. We show that $\mu \simeq v$. In fact, first we note that $T_{f} v=\mu_{\omega}^{f}$. Next, for any $A \in \mathfrak{B}$, there exist some countably-infinite orthonormal system $f_{1}, f_{2}, \ldots, f_{n}, \ldots$ and $\tilde{A} \in \mathfrak{B}\left(\mathbb{R}^{\infty}\right)$ such that $\chi_{A}(x)=\chi_{\tilde{A}}\left(\left(x\left(f_{1}\right), \ldots, x\left(f_{n}\right), \ldots\right)\right)$ for all $x \in H^{a}$. It follows that $v(A)=0 \Leftrightarrow \mu_{\omega}^{f}(\tilde{A})=0 \Leftrightarrow \mu^{f}(\tilde{A})=0 \Leftrightarrow \mu(A)=0$. Thus, 
Theorem 2. For any rotationally-quasi-invariant probability measure $\mu$ on $\left(H^{a}, \mathfrak{B}\right)$, there exists a rotationally-invariant probability measure $v$ such that $\mu \simeq v$. The explicit form of $v$ is as follows. $\quad v(B)=\int_{[0, \infty)} G_{v}(B) d P(v)$ for all $B \in \mathfrak{B}$, where $G_{v}$ is a canonical Gaussian measure on $\left(H^{a}, \mathfrak{B}\right)$ with mean 0 and variance $v$, and $P$ is a probability measure on $[0, \infty)$ defined by $P(E)=$ $\mu\left(x \in H^{a} \mid \varlimsup_{N} \frac{1}{N} \sum_{n=1}^{N} x\left(f_{n}\right)^{2} \in E\right)$ for Borel sets $E \subset \boldsymbol{R}$, using a countablyinfinite orthonormal system $f_{1}, f_{2}, \ldots, f_{n}, \ldots$ on $H$.

\section{References}

[1] Shimomura, H., Ergodic decomposition of quasi-invariant measures, Publ. RIMS, Kyoto Univ., 14 (1978), 359-381.

[2] Umemura, Y., Rotationally invariant measures in the dual space of a nuclear space, Proc. Japan Acad., 38 (1962), 15-17.

[3] — Measures on infinite dimensional vector spaces, Publ. RIMS, Kyoto Univ. 1 (1965), 1-47.

[4] Yamasaki, Y., Measures on infinite-dimensional spaces, Kinokuniya, 1978, in Japanese. 